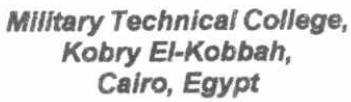

$9^{\text {th }}$ International Conference

On Aerospace Sciences \&

Aviation Technology

\title{
CFD INVESTIGATION OF AIRFLOW IN A CHAMBER UNDER DIFFERENT AIR SPEED, ASPECT RATIO, AND OUTLET LOCATION USING EXPLICIT MACCORMACK SCHEME
}

\author{
AHMED* A. A. SALMAN
}

\begin{abstract}
This paper aims at predicting the airflow patterns in a rectangular chamber under different inflow air speeds. This study ernploys a finite difference method with uniform single zone Cartesian grid to predict these airflow patterns. The governing equations are solved using an explicit MacCormack code developed by the author with forward and backward differences for predictor and corrector steps respectively. Special attention is paid to the boundary conditions that are strongly affected by air speed and openings. To stabilize the solution, a dissipation term is applied as a damping function to prevent divergence. Computations have been made on an isolated chamber of various rectangular shapes (i.e., different aspect ratios) and different inflow velocity profiles (i.e., different ventilation rates). Moreover, five different exhaust vent positions are considered. The locations of these outlet vents are on the left side wall as well as on the right side wall. This means that five different kinds of air diffusion models are studied. Fine and coarse grids are also considered for the analysis of patterns. Three grid distributions are studied for this purpose

The current algorithm has been successfully used for solving very low Mach number flows. This work is concerned with the steady state two-dimensional isothermal flow. A complete computed field is presented and discussed using an air flow velocity vector, streamlines and velocity ratios. The prediction of air movernent and airflow distribution inside the chambers has been investigated. Prediction of the velocity fields as well as the primary recirculation of indoor airflow showed anticipated results. The effects of ventilation inlet/outlet arrangements on air movement have been investigated. In the case of low-speed inflow, flow separation after the inlet and before the outlet(s) of the chamber has been captured using both the fine and coarse grids. In case of high-speed flow, only one big circulation has been captured.
\end{abstract}

\section{KEYWORDS}

Cartesian coordinates; Airfiow pattern; Explicit finite difference; Streamlines trace; Velocity vectors; MacCormack scheme. 


\section{INTRODUCTION}

\subsection{Computational Work}

Over the last few years, considerable developments in the area of Computational Fluid Dynamics (CFD) have been made. Calculation of flow problems is always bound to numerical methods except for simple situations. These numerical methods can be based on finite differences, finite volumes, finite element, etc. A wide range of methods of varying complexity has been developed with no single method that is universally appropriate. The selection of method varies according to the required level of accuracy, the availability of data and the type of geometry under consideration. For the determination of the airflow characteristics, two methods can be used. The first method is an explicit method in which all the input data are known by carrying out the calculation procedures step by step. The second method is the implicit method. This method is based on a single zone model using conservative flow equations that have to be solved iteratively for the unknowns.

Baskaran, et. al. [1] review the state-of-the art in computational, including finite element, finite difference methods and control volume for wind engineering problems. Analyzing and predicting the details of natural ventilation and airflow patterns, within individual rooms have been studied by many authors $[2,3$ \& 4] using CFD models. Rehm, et. al. [2] used Large Eddy Simulation algorithm (LES) to compute surface pressure distributions on an isolated building of various rectangular shapes. Emmerich [3] used a LES model to analyze building Indoor Air Quality (IAQ) and ventilation systems. Negrao [4] modeled indoor air flows encountered within buildings using Environment System Performance; $r$ for research orientation (ESP-r) CFD simulator. Clarke, et. al. [5] described the implementation of a computational fluid dynamic algorithm within the ESP-r system. Ref. [6] gives a very useful collection of information regarding natural ventilation in buildings with design recommendations. Variety of building configurations with different aspect ratios and modeling wind conditions around a group of buildings has been considered. The performance of a ventilation system for improving indoor air quality of the workshop has been studied numerically by Peng and Davidson [7], Svidt, et. al. [8], and Svidt and Bjerg [9]. The former studied the effect of room geometry and heat sources in the design of ventilation system. They used an air flow rate of $375 \mathrm{~m} 3 / \mathrm{h}$ at $1.9 \mathrm{~m} / \mathrm{s}$ and a temperature of $10^{\circ} \mathrm{c}$ below the room temperature. One big recirculation that occupied the whole zone has been observed. A study of low-Reynolds number effects in backward-facing step flows using LES scheme has been reviewed by Davidson and Nielsen [10]. They stated that the flow in ventilated rooms is often not fully turbulent, but in some regions the flow can be laminar. They showed that the LES scheme could predict the recirculation region properly for high Reynolds number rather than for small value. Johansson and Davidson [11] used a multigrid method in a 3-D SIMPLEC code to study both turbulent and laminar flow for a 2-D and 3-D ventilated enclosure. Davidson and Nielsen [12] and Davidson [13] used LES with an explicit finite volume, central difference method to predict the recirculating flow in a 3-D ventilated room. Jones, et. al. [14] summarized the principles of natural ventilation for improving livestock buildings. Emmerich and McGrattan [15] described the application of LES model to model ventilation airflow in a 3-D room. Their results were in good agreement with measured values. However, agreement was not good near the floor and ceiling. They said that this disagreement is due to Sagorinsky's constant and grid resolution. Feustel [16] developed an interzonal model (COMIS) to calculate air flows and pollutant transport mechanisms in both single and multizone buildings. Lu, et. al. [17] analyzed, computationally the air movement and aerosol particle distribution in a ventilated two-zone chamber with a small interzonal opening. 
Awbi [18] investigated the air movement and the distribution of $\mathrm{CO}_{2}$ in a natural office room and an atrium. Wind-driven and buoyancy-driven flows have been considered in his calculations. Morrison [19] used the adaptive conflation controller to manage all interactions between thermal and CFD modeling domains. These adaptive modeling techniques advance the modeling of indoor air flow and internal surface convection within whole-building simulation.

\subsection{Experimental Work}

Bjorn, et. al. [20] showed experimentally that the ventilation capacity, thermal comfort and indoor air quality can be improved by using different window types and windows in different positions in the natural ventilation system. Their results showed that different window opening strategies result in quite different airflow and thermal comfort conditions. Wind blowing against an endwall creates drafts if the air can enter the upwind end and travel the length of the building without obstructions. Awbi [21] discussed the parameters that should be considered in designing natural ventilation systems and presented a procedure for calculating the air flow rate due to wind and buoyancy. He used solar-induced ventilation as an application in ventilating commercial buildings. Loomans [22] measured data for air flow in an office room equipped with an air conditioning system. He used a helium-filled soap bubble to visualize the flow pattern. Temperature and velocity measurement data have been obtained. Zhao, et. al. [23] used a measurement technique based on Particle Image Velocimetry (PIV) to measure qualitative airflow patterns and distribution in ventilated airspaces. Martin, et. al. [24] measured the indoor air flow in the working area of machines for laser materials processing. The results serve as an aid in construction of ventilation systems in order to minimize workplace concentrations.

In Ref. [25] a comparison of the experimental and simulated flow behavior in room model were obtained. A reasonable prediction of the main recirculation flow and secondary flows has been obtained with course grid. Jaluria and Torrance [26] gave few practical problems for flow patterns of 2-D creeping flow, with inflow and outflow on the same side of a fluid tank as well as with infiow and outflow located on the opposite ends of a fluid tank.

\subsection{Current Work}

This study employs a finite difference method with uniform single zone Cartesian grid. The governing equations are solved using an explicit MacCormack code developed by the Author [27] with forward and backward differences for predictor and corrector steps respectively. The objective of this paper is to predict air flow in a rectangular chamber under different inflow air speed, aspect ratio, and outlet location. The effect of inlet velocity on the expected generated vortices has been analyzed. These vortices are generated when the flow interacts with the wall, floor, and ceiling or when two opposite vortices interact with each other. It was found that the instantaneous fluctuations were very large, for example, in the region of the wall or intermediate jet near the walls or in the middle of chamber. It was found that the results were dependent on the dissipation constant. Therefore, in this paper, the numerical dissipation term has been fixed for all study cases to achieve numerical stability and consistency. 


\section{GOVERNING EQUATIONS}

The equations below are a conservative form of Euler equations for 2-D compressible flow.

Conservation of mass

$\frac{\partial \rho}{\partial t}+\frac{\partial \rho u}{\partial x}+\frac{\partial \rho v}{\partial y}=0.0$

Conservation of $x$-momentum

$$
\frac{\partial \rho u}{\partial t}+\frac{\partial\left(P+\rho u^{2}\right)}{\partial x}+\frac{\partial \rho u v}{\partial y}=0.0
$$

Conservation of $y$-momentum

$$
\frac{\partial \rho v}{\partial t}+\frac{\partial \rho u v}{\partial x}+\frac{\partial\left(P+\rho v^{2}\right)}{\partial y}=0.0
$$

Conservation of energy

$$
\frac{\partial \rho e}{\partial t}+\frac{\partial(P+\rho e) u}{\partial x}+\frac{\partial(P+\rho e) v}{\partial y}=0.0
$$

In the above equations, $\rho$ is the density, $u$ and $v$ are the flow velocities in the $x$ and $y$ directions respectively, $p$ is the pressure, $t$ is the time, and $e$ is the total energy per unit mass. For an ideal gas, the pressure is related to the total energy per unit mass by:

$$
p=(\gamma-1)\left[\rho e-0.5 \rho\left(u^{2}+v^{2}\right)\right]
$$

Where the specific heat ratio $(\gamma=1.4)$ is constant for all calculations.

For incompressible flow, it follows from the above equations that the density field will be "frozen", i.e. kept constant. The above equations are solved by discretizing the equations using finite difference technique. Performing a direct numerical simulation (DNS) that involves solving these equations directly for the airflow characteristics.

All dimensionless, the lengths relative to a length scale $L$ determined by the height of the chamber, the velocity relative to a scale $\mathrm{V}_{0}$ determined by the incoming flow, the pressure relative to the dynamic pressure $\rho_{0} \mathrm{~V}_{0}{ }^{2} / 2$ and the time relative to the length scale and the velocity scale. $P_{0}$ is the ambient pressure, $\rho_{0}$ is the ambient density, and $V_{0}$ is the freestream uniform air flow velocity. The computations reported here were performed on one processor of a $650 \mathrm{MHz}$ with $20.5 \mathrm{~GB}$ memory.

\section{NUMERICAL TECHNIQUES}

Since the flowfield is characterized by constant density, pseudo incompressible model was applied. In the code, the density is relaxed and kept constant as free stream value after each iteration. This condition must be applied to satisfy the continuity equation for incompressible flow. Computational domains covering different areas are given in Tables 1,2, and 3. It is normal to employ some form of dissipation terms in order to obtain a good converged solution and to avoid nonphysical oscillations. The specification of dissipation terms depends on the user experience. A third order pressure dissipation is introduced into the program. The details of this dissipation were indicated in Ref [27]. A uniform Cartesian grid was adopted. A second order MacCormack scheme with forward prediction and backward 
correction methods was applied. This scheme has been used by the Author [27] and by Wendt [28] on a uniform grid throughout the physical space. In combination with the rectangular geometry already existing in the physical plane this means that there is no grid transformation.

The solution procedure can be summarized as follows:

1- Solve for the predictor values $q_{p t}, q_{p 2}, q_{p 3}$, and $q_{p 4}$ corresponding to $\rho, \rho u, \rho v$, and $\rho e$.

2- Calculate the pressure from the equation of state based on the above values.

3- Find $\Delta q_{p}$ at each cell.

4- Impose the predicted boundary conditions.

5- Solve for the corrector values $q_{c 1}, q_{\mathrm{c} 2}, q_{\mathrm{c} 3}$, and $q_{c 4}$ corresponding to $\rho, \rho u, \rho v$, and $\rho e$.

6- Calculate the pressure from the equation of state based on the above values.

7- Find $\Delta q_{c}$ at each cell.

8- Calculate the new values of $q_{1}, q_{2}, q_{3}, q_{4}$, and $P$.

9- Set the density equal to the free stream value.

10-Impose the corrector boundary conditions.

11-Check the convergence condition. If it converges, printout the results.

$12-\mathrm{Go}$ to the next time step and repeat step 1 to 9 and continue

\section{BOUNDARY AND INITIAL CONDITIONS}

Boundary conditions are explicitly implemented with inflow-outflow conditions and solid boundary conditions.

4.1. Inflow Boundary

The magnitude and direction of uniform velocity profile must be specified at the inlet, i.e. $u=U_{\text {in }}$ and $v=0.0$. The ambient values of pressure, $P_{0}$ and density, $\rho_{0}$ are considered.

\subsection{Outflow Boundary}

The entire outlet velocity profile was scaled from the inlet velocity profile by the opening length ratio to provide a nominal conservation of mass, i.e. global continuity was taken as constant over the inlet and outlet(s). The cutlet pressure profile, vertical velocity component and density are extrapolated from interior domain using Numann conditions, i.e. zero gradient boundary conditions were specified.

\subsection{Solid Boundary}

For our computations, the second normal derivative for the pressure is set to zero at all solid boundaries. Free-slip boundary conditions were chosen for the ceiling and the floor as well as for the right and left side walls of the chamber. Impermeability condition is used for the normal velocity to the solid surface, i.e.

For right and left side walls, $u=0$ and $\frac{\partial v}{\partial y}=0.0$ and

For the ceiling and the roof, $v=0$ and $\frac{\partial u}{\partial x}=0 \Omega$

\subsection{Initial Conditions}

The air inlet velocity is adopted to be uniform over the inlet section. The pseudo time marching calculations are started with the air inside the chamber at rest, i.e. $u=v=$ 0 . Adiabatic boundary conditions were imposed on all walls. 


\section{RESULTS OF CASE STUDIES}

Numerical solutions for the prediction of the flow patterns are presented in this section. The present approach, based on an explicit finite-difference scheme, handles any geometry that can be represented on a rectangular grid. The program works mainly steady state simulations. However some instantaneous simulations were performed in order to evaluate the propagation of the air stream and formulation of vortices. The Air was supplied from a high-level slot adjacent to the ceiling with a fixed height and exhausted through either one or two vents. Air flow simulation inside a chamber of four different aspect ratios were reported. Two different uniform incoming speeds were investigated. These simulations were performed using coarse and fine grid distributions.

One coarse grid case has been compared with Negrao's work [4] to check the validity of the code. The favorable agreement arising from these comparisons justify the use of this scheme for additional computations. Case 2 and 3 are sirnilar in their rectangular shape and aspect ratios but they are different in the inflow air speed. Calculations were performed for each of these geometries at inflow speeds 0.455 and $2.275 \mathrm{~m} / \mathrm{s}$ corresponding to 0.00132 and 0.00660 Mach numbers (based on speed of sound $=345 \mathrm{~m} / \mathrm{s}$ ) respectively. In Case 4 , a chamber with $A R=1$ was considered, information about siting of one air inlet in relation to different air outlet siting locations has been studied. In Case 5 the inlet velocity and the input and the cutput locations are kept constant while three levels of grid refinement were considered.

The samples of Convergence history based on the log of residual error of the pressure for some cases are given in Fig. 1. It clearly shows that steady state solution has been achieved with aimost five order of magnitudes convergence after long period of pseudo time.

\subsection{Test Case One}

This case was used as a test case at which air is supplied from one inlet slot of 0.15 $\mathrm{m}$ height on the upper left-corner of the side wall and exhausted from one outiet of $0.45-\mathrm{m}$ on the lower right corner of the side wall. An inlet air velocity was chosen to be $0.51 \mathrm{~m} / \mathrm{s}$. A uniform grid of $21 \times 21$ grid points was used. Velocity vectors, streamlines and velocity profiles, $u / U$ in, at $x=1, x=2$ (vertical plane positions) and closer to both the ceiling and floor (horizontal plane positions) are shown in Fig 2. All flow patterns have been obtained with one big flow circulation inside the chamber. However, small discrepancy between our results and Negrao's results [4], in the magnitude of the velocity vectors and the location of the recirculation center, has been observed. This discrepancy was expected because the type of flow (inviscid with dissipation term rather than viscous with turbulence models), grid distribution (uniform rather than non-uniform one) and outflow boundary conditions.

\subsection{Case Two; Inflow Velocity Equal $0.455 \mathrm{~m} / \mathrm{s}$}

Figure 3 shows sample of the computational Cartesian grid used in this case for aspect ratio equal 3 with grid points equal $91^{\star} 31$ (plotting every other point). Figure 4 shows some snapshots, at different instants, of the primary calculating velocity vectors generated as the flow enters the chamber.

Table 1. Grid points and aspect ratio used in case 1

\begin{tabular}{|c|l|c|c|c|}
\hline Case No. & Location of inlet and outlet vents & Grid points & Aspect Ratio (AR) \\
\hline A & One inlet on the upper-left & $17^{*} 31$ & $1 / 2$ \\
\cline { 4 - 5 } B & corner and one outlet on the & $31^{\star} 31$ & 1 \\
\cline { 4 - 5 } & lower-right corner of the side & $61^{\star} 31$ & 2 \\
\hline w & walls & $91^{\star} 31$ & 3 \\
\hline D & & & \\
\hline
\end{tabular}


The specification of grid points and aspect ratios used in this case are given in Table 1. Figure 5 shows the computed streamlines plot within different chambers of different aspect ratios. Air was fed in from one inlet slot of $0.15-\mathrm{m}$ height with very low velocity, $0.455 \mathrm{~m} / \mathrm{s}$ corresponding to 0.00132 Mach number. Air was extracted from inside the chamber through one outlet vent of the same height as the inlet. In this case, an equal impact on the flow has been obtained as the suction beneath the ceiling. Calculations showed that two opposite recirculation bubbles were generated. The first one was located after the inlet and closer to the floor, while the second was located before the exit and closer to the ceiling. The flow is unstable and takes long time to reach steady state. This is related to the low flow velocity [27]. A marked upward flow along both the right and the left walls was identified. This is because the circulation of the generated bubbles was in the opposite directions. The streamlines, in addition to showing the direction of flow, indicate changes in speed. The closer they are crowded together, the greater is the speed at that section because the same amount of fluid must pass between two streamlines at any section. Figure 6 shows velocity vector fields for this case. For all aspect ratio, one can observe development of qualitatively similar flow patterns with a double-vortex circulation separated by a speed-jet. The intensity of this speed-jet decrease as it moves downstream towards the outlet position. However, quantitative differences are significant for different aspect ratio (AR). While increasing the chamber aspect ratio (width/height), the upper vortex moves closer to the ceiling with less intensity and the intensity of the lower vortex is getting higher. For $A R=1$, this phenomenon of dividing the chamber with a high-speed jet is notable. It is interesting to note for this case, the within-jet velocity keeps high value in the middle of the chamber. It is similar to the internal gear compressor characteristic. The length of the velocity vector increases because the direction of both the upper and the lower vortices is compiled together. In this flow patterns, the near-floor velocities are less than the near-ceiling velocities. Finally, as it was observed by Ref. [24], this low volume flow rate of the fresh air causes a high turbulence/ and or instability of the indoor air flow. The size of the predicted recirculation bubble near the ceiling and below the inlet is getting larger as the aspect ratio increases.

\subsection{Case Three; Inflow Velocity Equal $2.275 \mathrm{~m} / \mathrm{s}$}

Figures 7 and 8 show streamlines and velocity vector fields for this case. From these figures and for all the aspect ratios, one can observe the development of qualitatively similar flow patterns with a single-vortex circulation in the middle of the chamber However, quantitative differences are significant. The center of circulation is below the middle of the side wall because the speed-jet is stronger and closer to the ceiling rather than to the floor for AR less than or equals 2. For AR equals 3 , the center of the circulation is almost at the center of the chamber. With an increase in the chamber aspect ratio (width/height), the central low-speed region decreases and spreads almost two-third of the chamber. For all aspect ratio, the flow is divided into a near wall high-speed jet and a relatively low-speed circulation flow. It is interesting to note that the high-speed jet keeps high value up to the exit vent. The length of the velocity vectors increases to about three times the inlet velocity vector in some cases, such as the $A R=1$ and low speed inflow case.

Comparing the internal flow patterns for case 2 and 3 , one can see that they are completely different. When the inflow velocity increases, the flow presents have one circulation inside the chamber, which is not evident when the inflow velocity decreases. On the other hand, a recirculation flow in the upper part of the chamber is evident when the inflow velocity is decreased. Although the two recirculating flows 
occur in opposite direction, both help to increase the mass flow rate and consequently the jet flow speed.

Results for the distribution of the horizontal velocity profiles, normalized through the inflow free stream velocity $U_{\text {in, }}$ at the ceiling and floor of the chamber for inlet velocities, namely 0.455 and $2.275 \mathrm{~m} / \mathrm{s}$ at different aspect ratios are shown in Figure 9. In these cases, the outlet velocity is kept as the inlet velocity. The effect of recirculation zone on the velocity profile is observed

For low-speed flow (on the left side): The velocity profiles, on the floor (solid line), started at zero value and increased negatively to its maximum value and then decreased towards the zero value (reattachment point) and then increased positively along the chamber until the exit value. This reattachment point depends upon the recirculation zone location and its strength closer to the floor. Large differences between the predicted values for different $A R$ have been observed On the ceiling (dashed line), opposite situations are observed. In this case, the ceiling velocity decreases as the reattachment point is approached and after that it decreases. The velocity profiles for either the floor or the ceiling, for all aspect ratios, were not symmetric with the exception of $A R=1$.

For high-speed flow (on the right side): The velocity profiles show small. differences between the predicted values as the AR increased. This is because there. is only one big recirculation zone located in the whole chamber. In this case, the predicted profiles in the recirculating flow show the reversed flow near the floor. This. reversed flow is going to return to a fully developed positive profile. The velocity profile on the ceiling increased, remained constant and after which it decreased back to zero value as the flow approached the upper right corner. However, on the floor, these curves are characterized by decreases and then they increased again to their value at the right side lower corner.

Comparisons of the results reveal a significant difference between the corresponding two cases due to the different in the inflow velocity conditions, the generated recirculation and its location. One can conclude that the size and location of the recirculation bubbles are sensitive to the value of inlet velocity as well as the outlet location.

\subsection{Case Four; Effect of Outlet Location(s) on the Predicted Flow Pattern}

Table 2. Position of the inlet and outlet locations

\begin{tabular}{|c|l|l|}
\hline Case No. & Inlet Location & Outlet Location (s) \\
\hline A & \multirow{2}{*}{ On the upper-left } & Upper-right corner of the side wall \\
\hline B & Middle of the right side wall \\
\hline C & corner of the side wail & Lower and upper corners of the right side wall \\
\hline D & & Lower-right corner of the side wall \\
\hline E & & Lower-left corner of the side wall \\
\hline
\end{tabular}

Specifications of the outlet location are indicated in Table 2 for five different models: For all models, the location of the inlet is fixed at the upper-left corner of the side wall. Figures 10 and 11 show the effect of changing the outlet location on streamlines and velocity vectors patterns inside a chamber of $A R=1$. One big circulation is observed for model-A and Model-C. For Model-B, -D and -E two zones of circulation are observed. These recirculation bubbles are almost symmetrical around the major chamber's diagonal for Model-D and -E. The size of the recirculation bubble below the inlet is almost generated, however the near ceiling and left side wall generated 
bubble depend on the outlet location. The size and location of these recirculation bubbles are sensitive to the outlet position.

5.5. Case Five; Effect of Grid Point Distribution on the Predicted Flow Pattern

Table 3. Effect of grid points distribution (grid refinements)

\begin{tabular}{|c|l|c|}
\hline $\begin{array}{c}\text { Case } \\
\text { No. }\end{array}$ & Location of inlet and outlet vents & Grid points \\
\hline 1 & Inlet from upper-left corner of the & $21 \times 21$ \\
\hline 2 & $\begin{array}{l}\text { side wall and outlet from lower-right } \\
\text { corner of the side wall }\end{array}$ & $35 \times 35$ \\
\hline 3 & & $41 \times 41$ \\
\hline
\end{tabular}

Figure 12 shows three levels of grid refinement used in our study as described in Table 3. The inlet velocity was $0.455 \mathrm{~m} / \mathrm{s}$ with a uniform profile. This effect of mesh size on the velocity vectors and streamlines distribution was considered. As it was expected, the computed streamlines using coarse and fine grids have yields close results for one aspect ratio chamber. However, a very little overall sensitivity to mesh size in the silence regions is observed. This little effect is clear in regions of large gradients where the finer mesh yields sharper circulation. Also, the created dead airspace region (minimum absolute velocity) is more concentrate in the middle of the recirculation bubble. The size of the predicted recirculation bubbles above and below the main diagonal between the inlet and outlet does not differ that much as the grid refinement increases.

\section{CONCLUSIONS AND FUTURE WORK}

An efficient explicit MacCormack algorithm is used to predict and compute the flow variables inside an isolated 2-D chamber of rectangular shape with different locations for inlet and outlet vents. For all the variants of the flow arrangement considered, a single-vortex circulation develops within the chamber for high inflow rate and double vortices for low inflow rate. Accordingly, when the positions of the vents are properly adjusted a proper air distribution between the inlet and the outlet can be obtained. Airflow simulation has been studied and here the findings are:

1- Steady state has been obtained using coarse and fine grids.

2- The location of both inlet and outlet vents has a great effect on the airflow velocity distribution inside the chamber.

3- Airflow patterns were greatly influenced by the arrangement of the outflow location.

4- The boundary conditions and the dissipation term could affect the numerical results (results of those effects not included in this work).

5- A sufficient high supply of fresh air is necessary to avoid partial internal recirculation and to minimize the degree of turbulence by vortices.

6- The central low-speed (silence) region increases as the inflow air speed decreased.

In the future studies, examination of internal/external flow in/over building shape with different roof slope will be considered. Moreover, the ratio of outlet to inlet opening sizes and the degree of obstruction to the flow either inside or outside the building will be investigated. The most important aspect for further study is the effect of dissipation terms on the CFD simulations. 


\section{REFERENCES}

[1] Baskaran, A., Csce, M., Stathopoulos, T. and Asce, F., "Prediction of wind effects on buildings using computational methods- State of the Art Review," Canadian Journal of Civil Engineering 21, pp. 805-822, (1994).

[2] Rehm, R. G., McGrattan, K. B., Baum, H. R. and Simiu, E., "An efficient Large Eddy Simulation algorithm for computational wind engineering: Application to surface pressure computations on a single building," NISTIR 6371, Building and Fire research Laboratory, Gaithersburg, MD 20899, August (1999).

[3] Emmerich, S. J., "Use of computational fluid dynamics analysis indoor air quality issues," NISTIR 5997, Building and Fire research Laboratory, Gaithersburg, MD 20899, April (1997).

[4] Negrao, C. O. R., "Conflation of computational fluid dynamics and building thermal simulation," Ph.D. Thesis, Department of Mech. Engineering, University of Strathclyde, Glasgow, UK, September (1995).

[5] Clarke, J. A., Dempster, W.M. and Negrao, C., "The implementation of a computational fluid dynamics within the ESP-r system," ESRU, Energy System Division, Faculty of Engineering, University of Strathclyde, Glasgow G1 1XJ, Brasilia, Brasil.

[6] "Natural ventilation in buildings" from Internet Links to:

http:/ffridge.arch.uwa.edu. au/local/natvent/NaturalVentilation/nBuildings.htm.

[7] Peng, S-H, and Davidson, L., "Performance evaluation of a displacement ventilation system for improving indoor air quality: A numerical study," Presented at the $8^{\text {th }}$. Int. Conf. on Indoor Air Quality and Climate, Edinburgh, Scotland, Vol. 2, pp.72-77, August $8^{\text {th }}-13^{\text {th }}$. (1999).

[8] Svidt, K., Zhang, G., and Bjerg, B., "CFD simulation of air velocity distribution in occupied livestock buildings," Proceeding of RoomVent'98, Sixth Int. Conf. on Air Distribution in Rooms, Stockholm, Sweden, pp. 491-496, (1998).

[9] Svidt, K., and Bjerg, B., "Computer prediction of air quality in livestock buildings," Indoor Environmental Engineering, Paper No. 56, ISSN 1395-7953 R9648, (1996).

[10] Davidson, L. and Nielsen, P.V., "A study of low-Reynolds numbers effects in backward-facing step flow using large eddy simulation," ROOMVENT'98, $6^{\text {th }}$. Int. Conf. on Air Distribution in Rooms, Vol. 1, pp. 125-132, Eds. E., Stockholm, (1988).

[11] Johansson, P. and Davidson, L., "A full multigrid method applied to turbulent flow using the SIMPLEC algorithm together with a collocated arrangement," Multigrid Methods IV, Birkhauser Verlag, pp. 245-256, (1994).

[12] Davidson, L. and Nielsen, P.V., "Large Eddy Simulation of the flow in a threedimensional ventilated room," $5^{\text {th }}$. Int. Conf. on Air Distributions in Rooms, ROOMVENT'96, Yokohama, Japan, Vol.2, pp. 161-168, July 17-19 (1996).

[13] Davidson, L., "Implementation of Large Eddy Simulation method applied to recirculating flow in a ventilated room," ISSU 1395-7953 R9611, Sweden, April 9 (1996).

[14] Jones, D. D., Friday, W. H. and DeForest, S. S., "Natural ventilation for livestock housing," from Internet Links to :http://www. agcom.purdue.edu/AgCom/Pubs/AE/AE97.html

[15] Emmerich, S. J. and McGrattan K. B., "Application of a Large Eddy simulation model to study room airflow," ASHRAE Transactions, Vol.104, Pt.1, February (1998). [16] Feustel, H. E., "COMIS-An international multizone airflow and contaminant transport model," Indoor Environment Department, Berkeley CA 94720, USA.

[17] Lu, W., Howarth, A. T., Adam, N., and Riffat, S. A., "Modeling and measurement of airflow and aerosol particle distribution in an ventilated two-zone chamber," Building and Environment, Vol. 31, No. 5 pp. 417-423, (1996). 
[18] Awbi, H. B., "Air movement in naturally-ventilated buildings," The University of Reading, Reading RG6AW, UK, (1996).

[19] Morrison, I. B-M. "The adaptive coupling of heat and air flow modeling within dynamic whole-building simulation," Ph.D. Thesis, Department of Mech. Engineering, University of Strathclyde, Glasgow, UK, May (2000).

[20] Bjorn, E., Jensen, J., Larsen, L., Nielsen, P.V., and Heiselberg, P., "Improvement of thermal Comfort in a naturaily ventilated office" proceeding of $21^{\text {st }}$. AIVC Conference, Netherlands, $26-29^{\text {th }}$ Sept. (2000).

[21] Awbi, H. B., "Design considerations for naturally ventilated buildings," Renewable Energy, Vol. 5, Part II, pp. 1081-1090, (1994).

[22] Loomans, M., "The measurement and simulation of indoor air flow," Ph.D. Thesis, University of Netherlands, (1998).

[23] Zhao, L., Zhang, Y., Wang, X., Riskowski, G. L., and Christianson, L. L., "Development of particle image velocimetry techniques for measurement of room airflow patterns in ventilated airspaces," From Internet links to: http://www. age. uiuc.edu/bee /RESEARCH /piv/ pivpaper2.htm

[24] Martin, D., Arndt, O. H., Ernst, W. K., Carsten, M. and Rolf, K., "Measurement of the indoor air flow in the working area of machines for laser materials processing," Industrial Safety Forum, Copenhagen'95, pp. 273-280, (1995).

[25] Fletcher, C. A. J., "Computational techniques for fluid dynamics 2," SpringerVerlag, (1987).

[26] Jaluria, Y. and Torrance, K. E., "Computational Heat transfer," Hemisphere Publishing Corporation, (1986).

[27] Salman, A. A., "The effect of changing channel passage area on the compressible flow characteristics with and without air injection," AEIC 2000 Meeting, Al-Azhar University, Cairo, Egypt, Sept. 1- 4 (2000).

[28] Wendt, J. F. with contributions by Anderson, J. D., Degrez, G., Dick, E., and Grundmann, R., "Computational Fluid Dynamics," ISBN 3-540-5947-X $2^{\text {nd }}$. Ed., Springer-Verlag, (1996). 

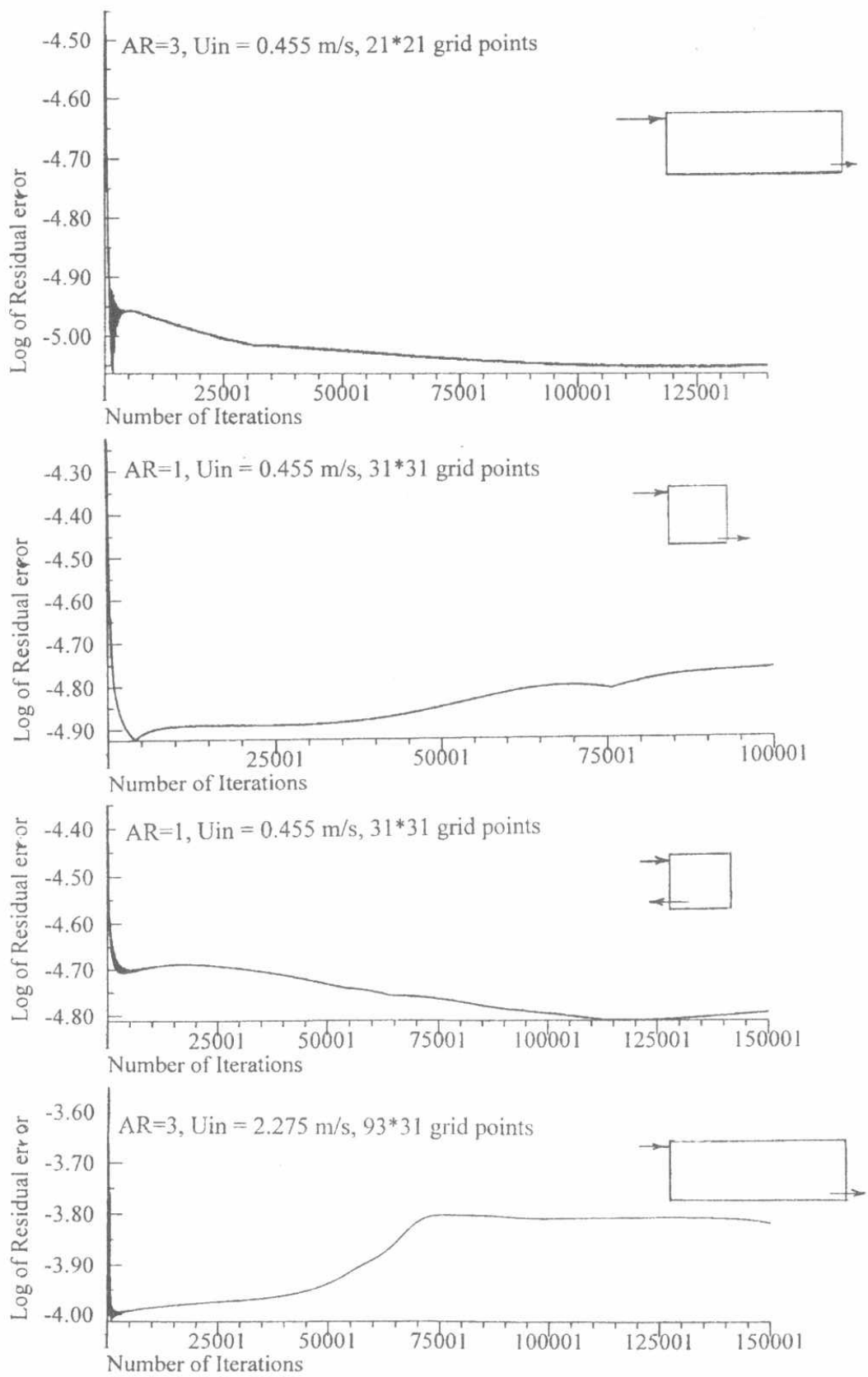

Figure 1 Sample of convergence history for different cases 


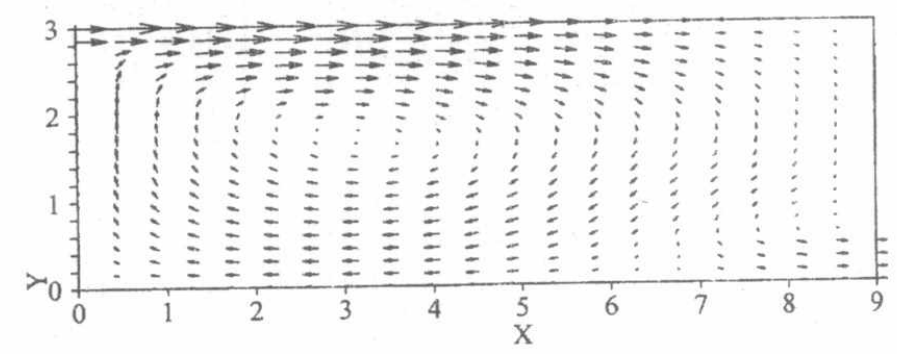

Computed velocity vectors
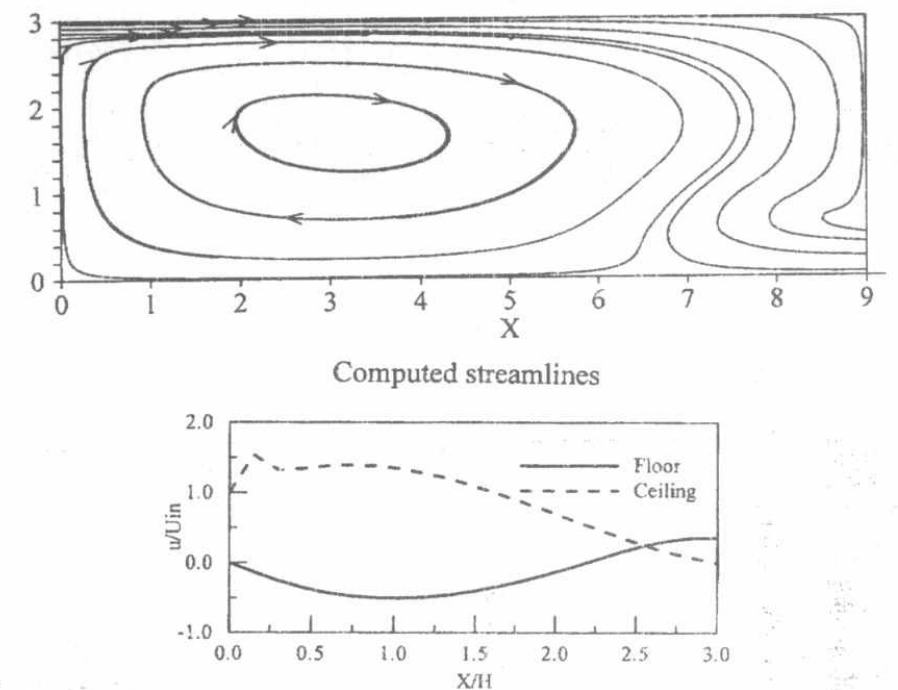

Velocity profiles over the floor and ceiling

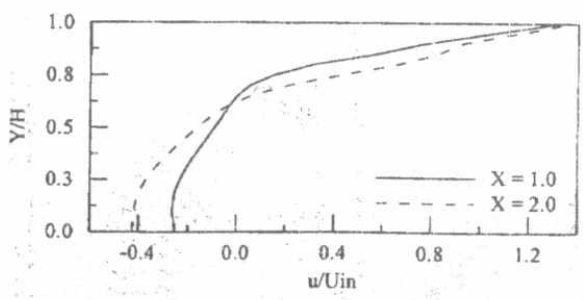

Velocity profiles at $\mathrm{x}=1$ and $\mathrm{x}=2$ down stream

Figure 2 Test case results, flow enters from the upper-left corner on the upward side wall while exhausted from the lower-right corner on the leeward side wall $\left(\Delta t=0.08, \varepsilon=0.0035, U_{\text {in }}=0.455 \mathrm{~m} / \mathrm{s}\right)$. 


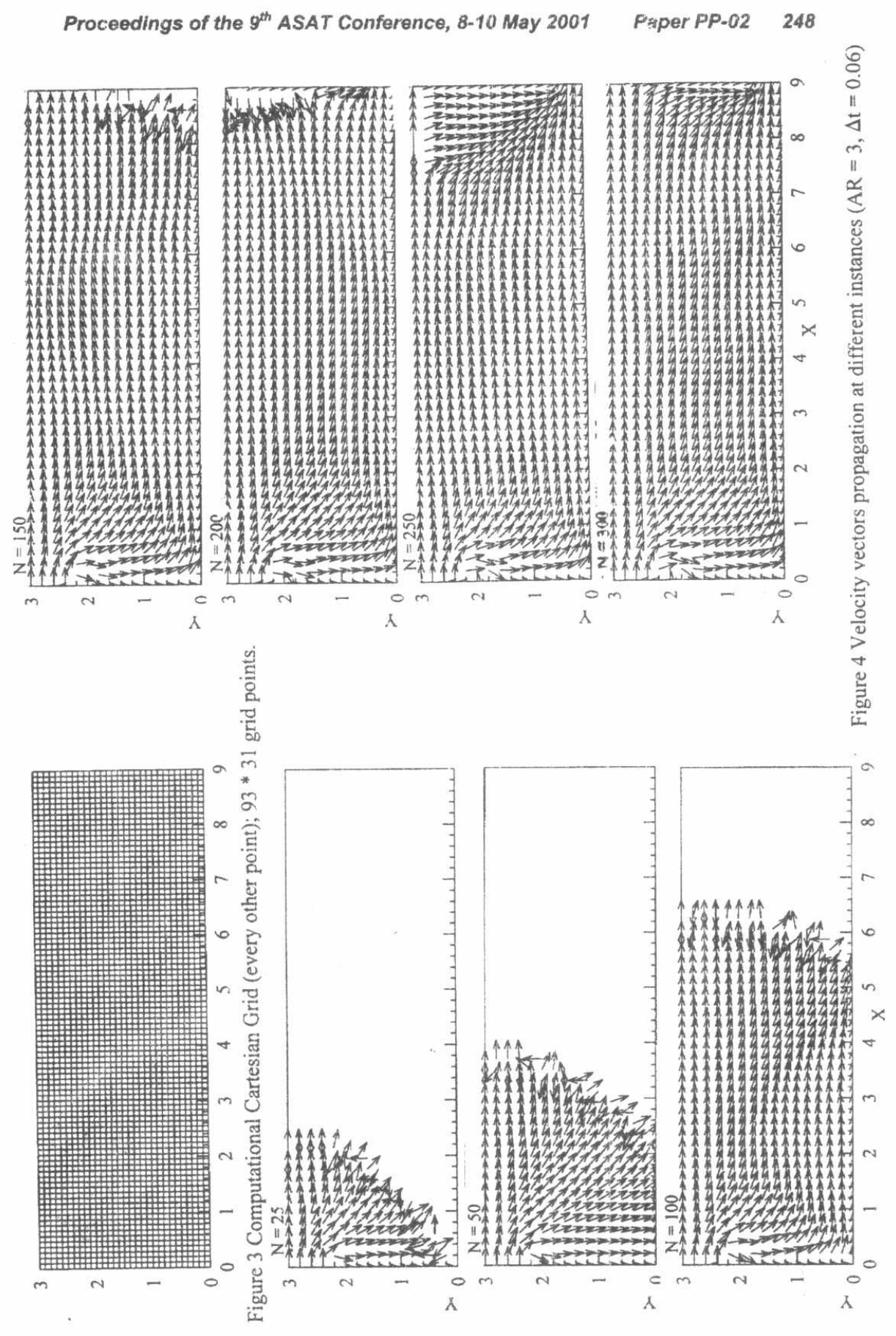



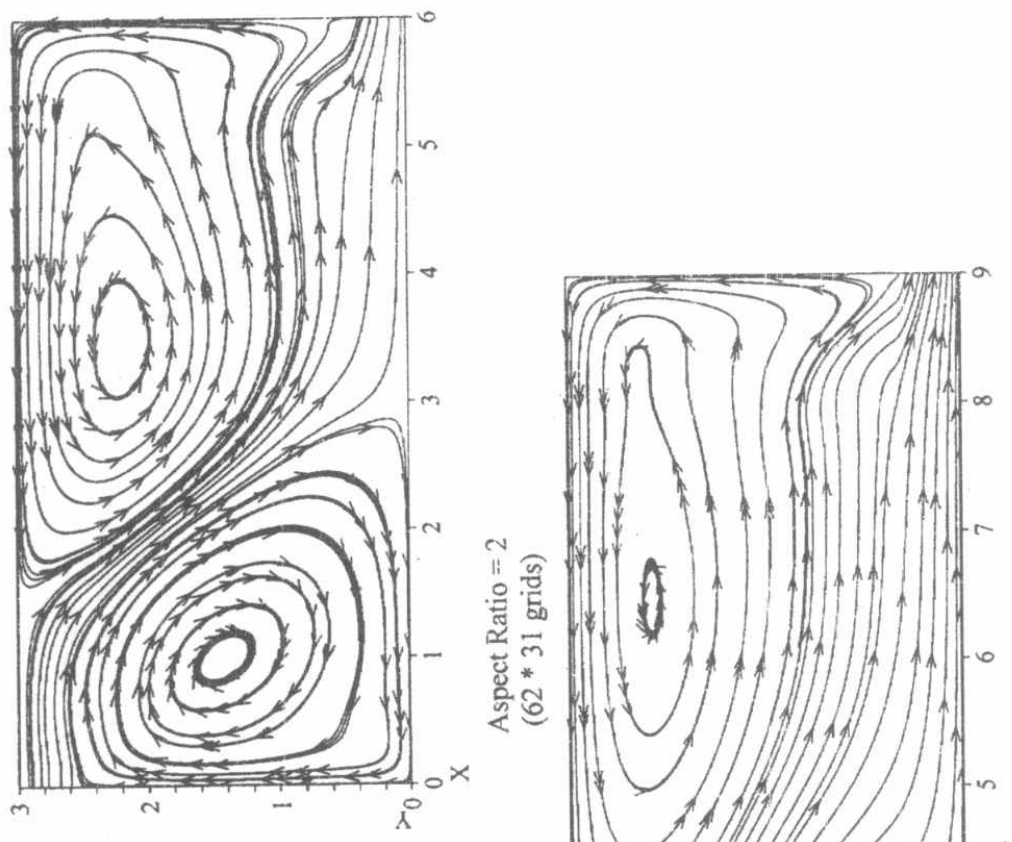

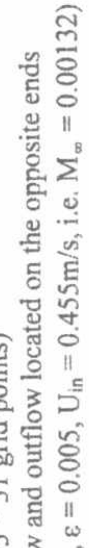
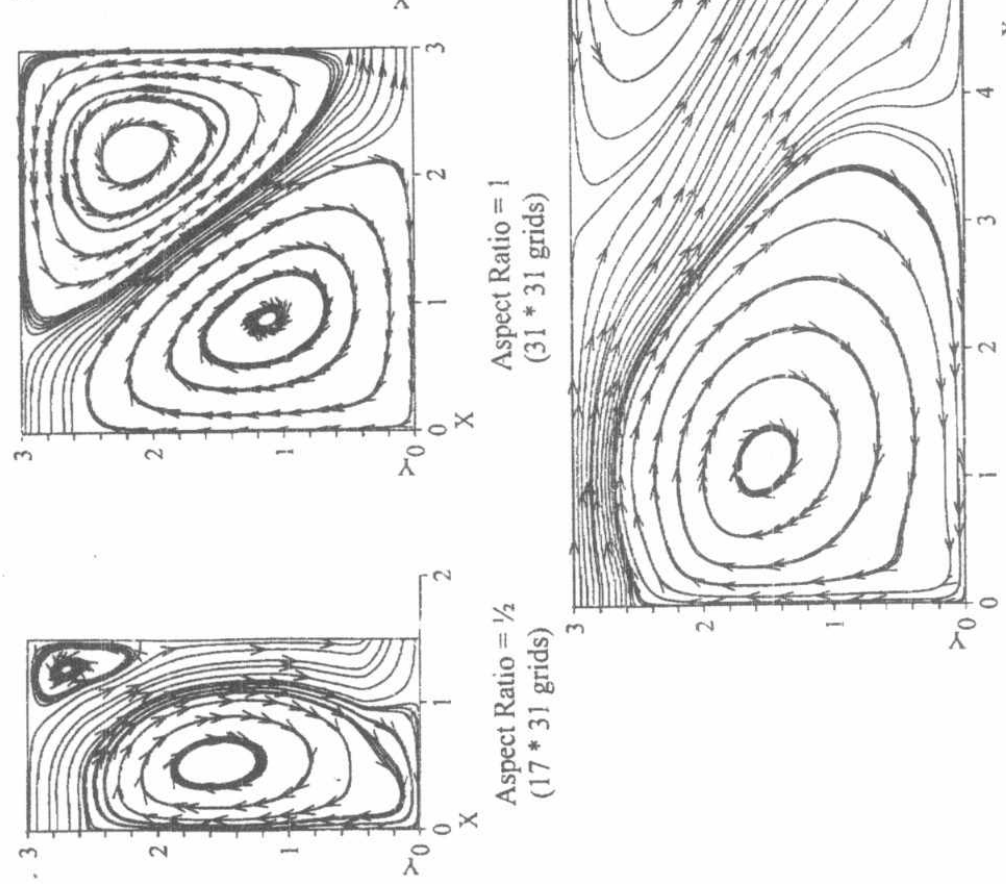

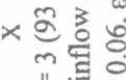

온

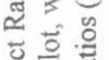

흠해

<总

ت

离

บ

范

羟

迅

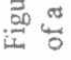



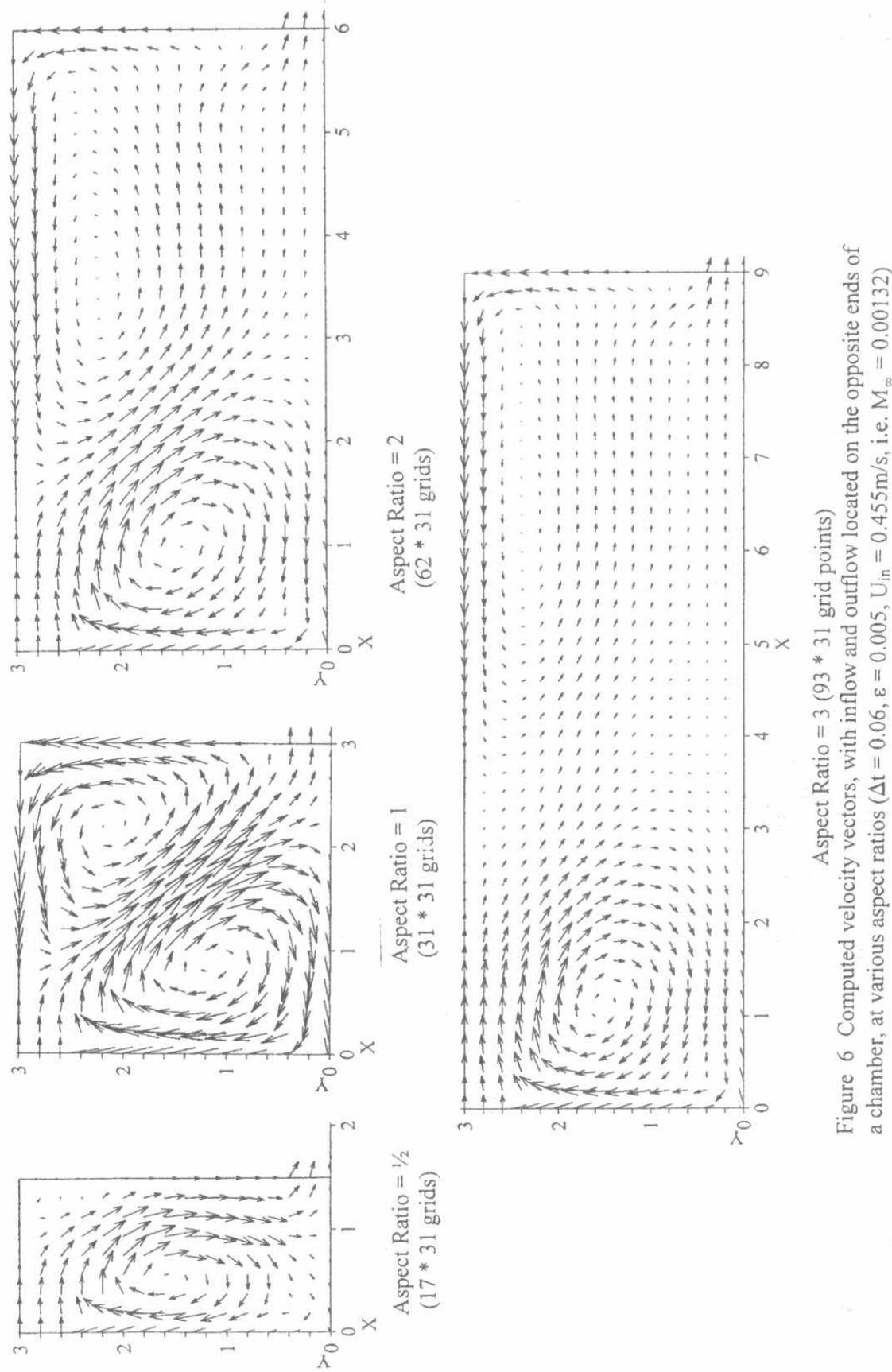

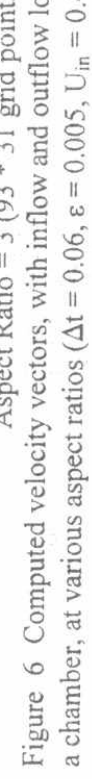

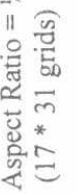



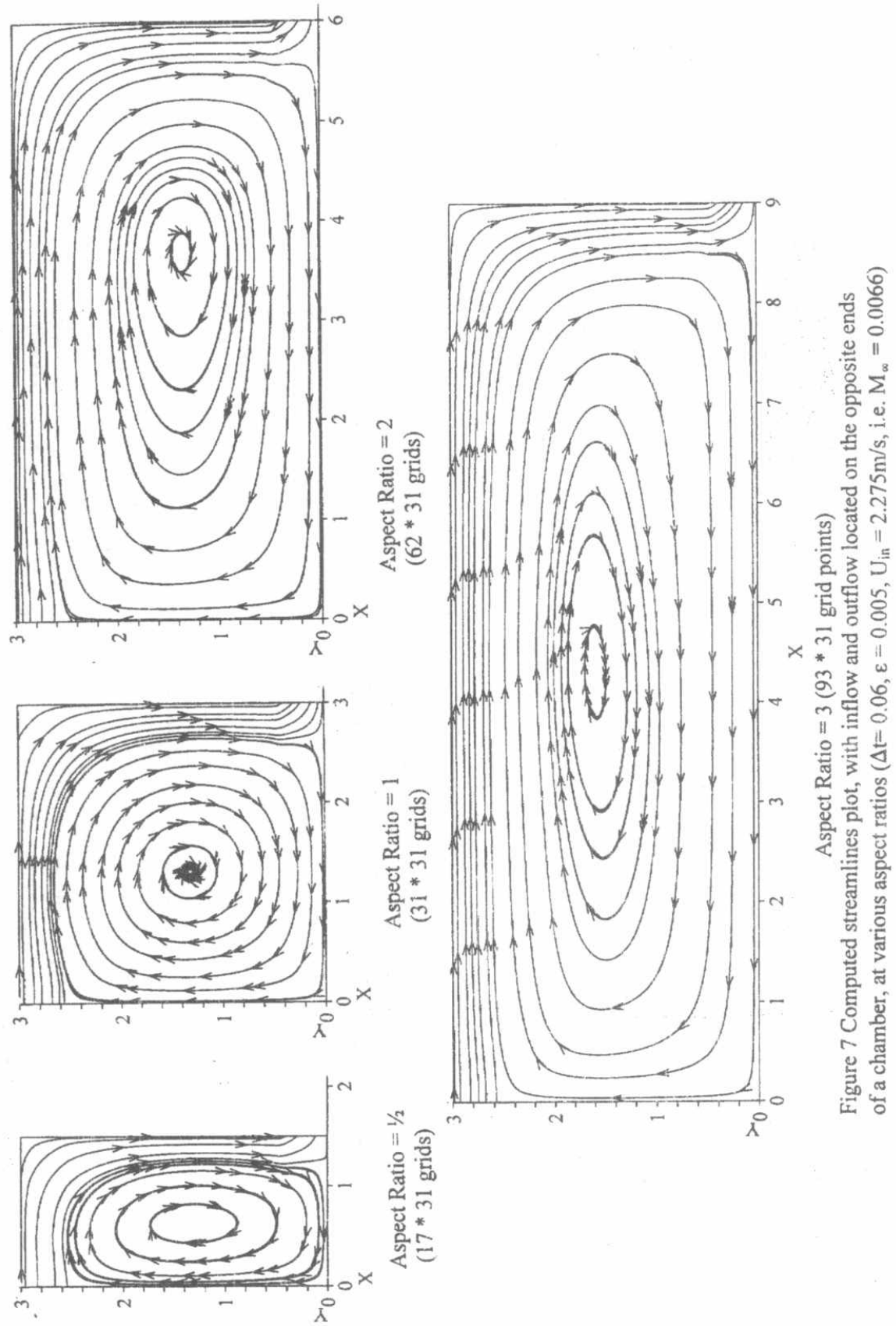

* है

융

铮过

홍. 응

总

⿷匚ّ

롱

过

놀 엉

鱼总

递泀 


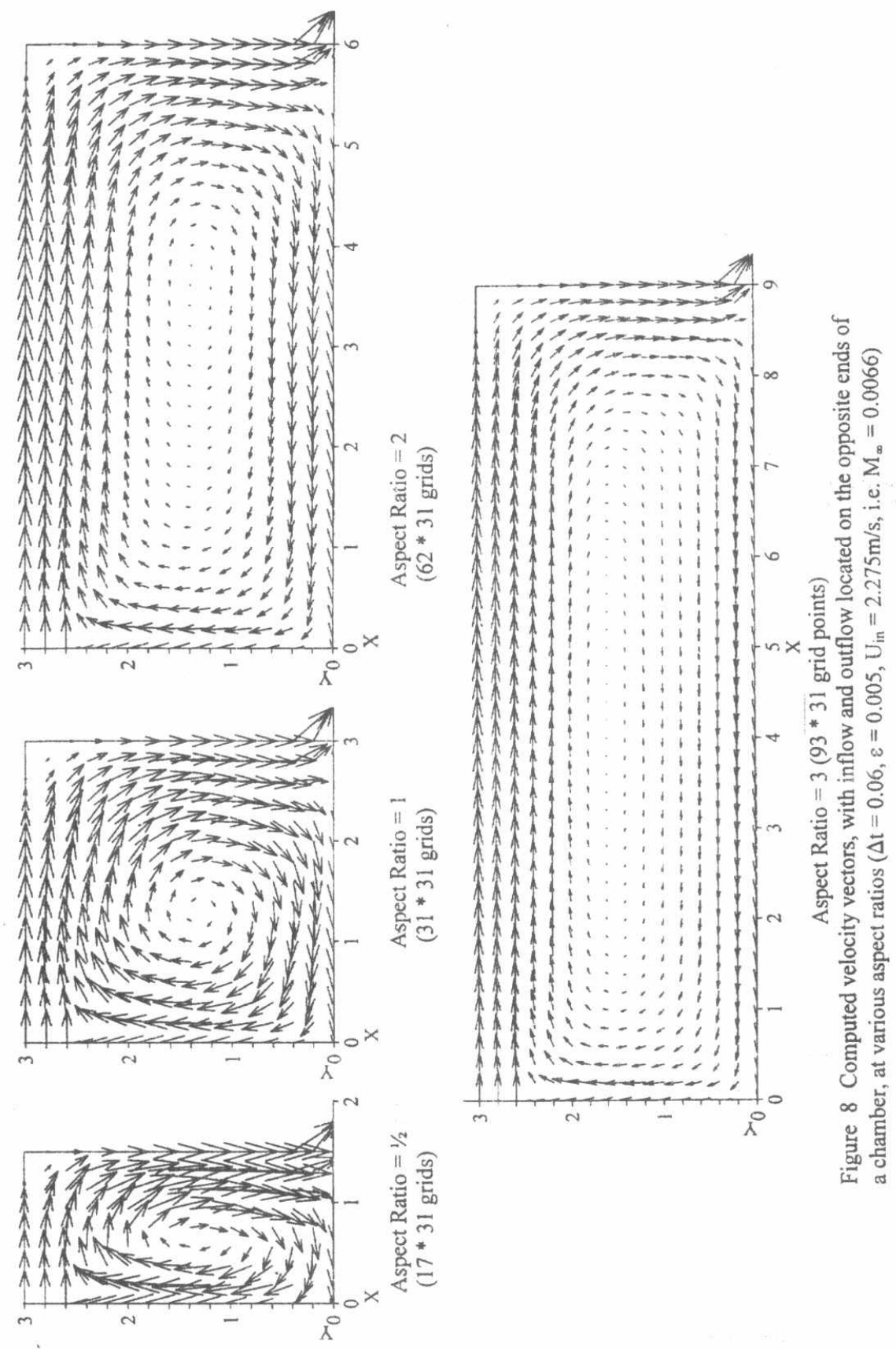



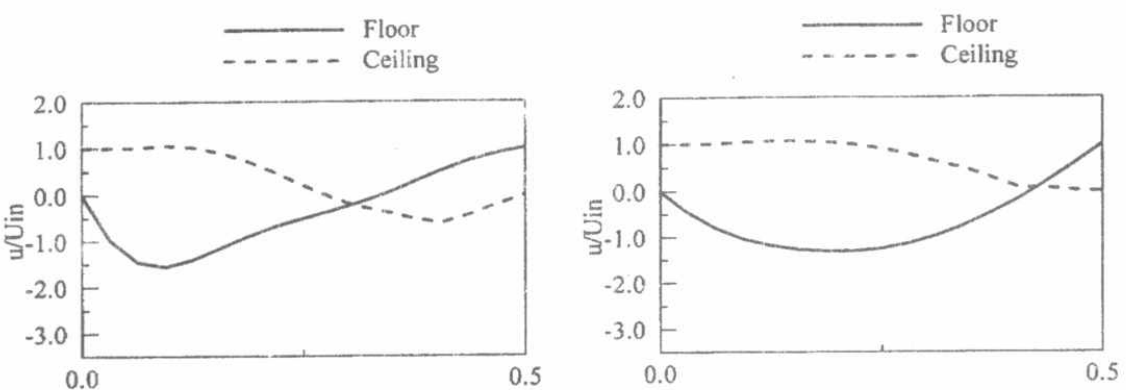

a) Aspect ratio $=1 / 2$
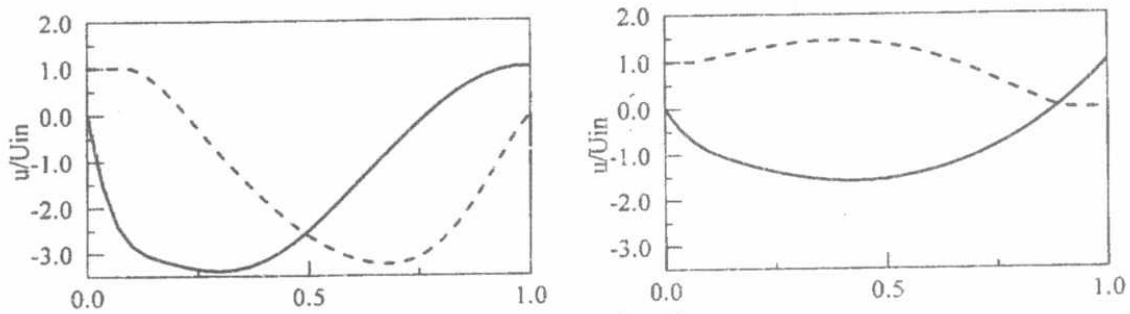

b) Aspect ratio $=1$
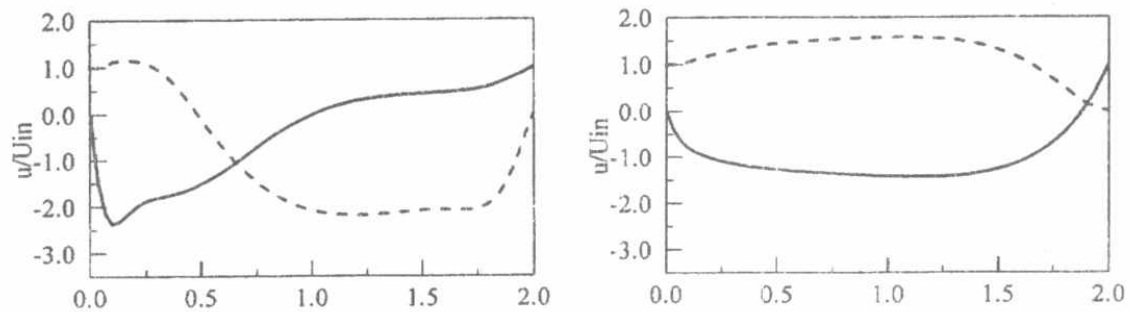

c) Aspect ratio $=2$
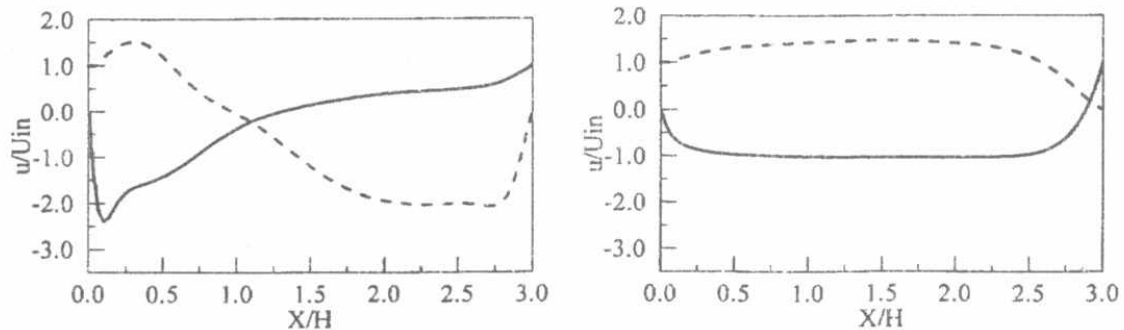

d) Aspect ratio $=3$

Low Speed, $\mathrm{U}_{\text {in }}=0.455 \mathrm{~m} / \mathrm{s}$

High Speed, $U_{\text {in }}=2.275 \mathrm{~m} / \mathrm{s}$

Figure 9 Effect of changing inflow velocity on the velocity profiles over the floor and ceiling of different chambers (different aspect ratios). 


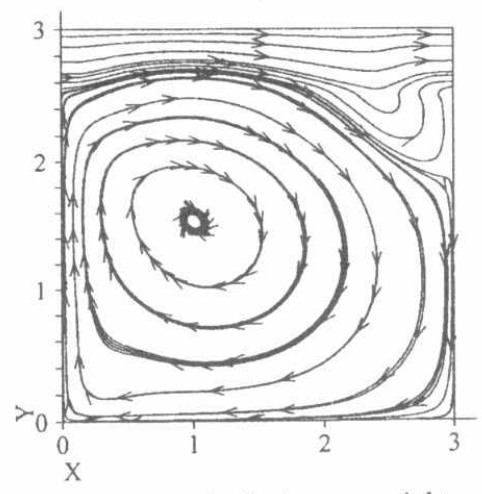

a) one outlet in the upper right corner of the leeward side wall

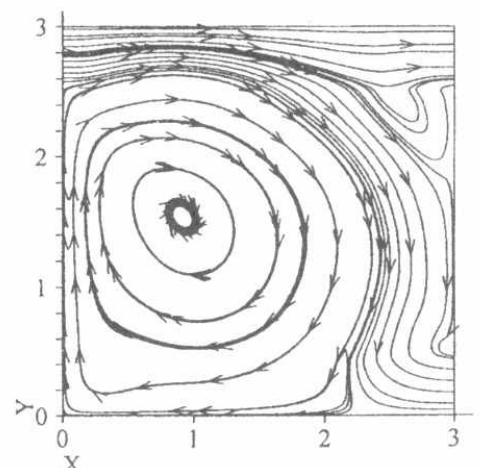

c) two outlets in the upper and lower right corners of the leeward side wall

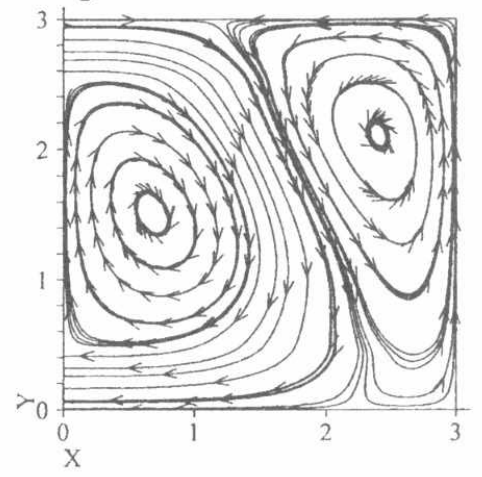

e) one outlet in the lower left corner of the upward side wall

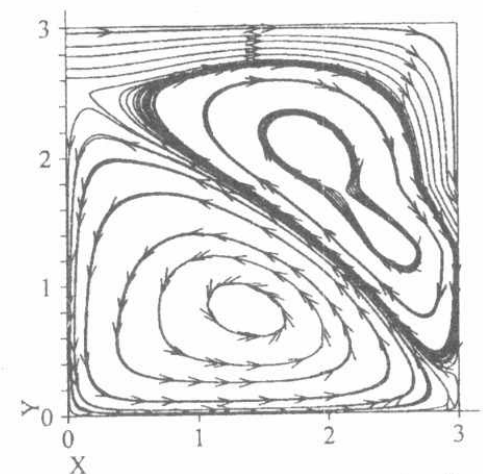

b) one outlet in the middle of the right leeward side wall

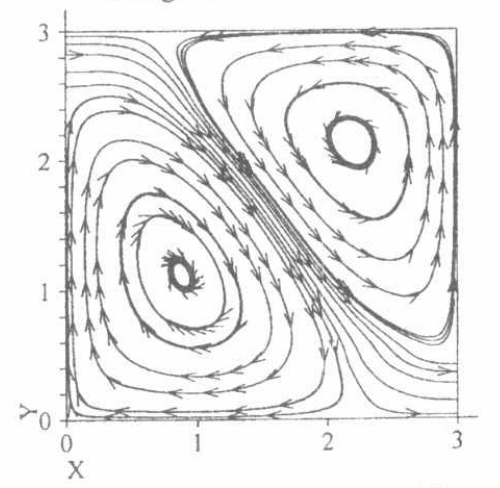

d) one outlet in the lower right corner of the leeward side wall
Figure 10 Effect of changing outlet location (s) on streamlines distribution inside chamber with $\mathrm{AR}=1$ $\left(\Delta t=0.06, \varepsilon=0.005, U_{\text {in }}=0.455 \mathrm{~m} / \mathrm{s}\right.$, i.e. $\left.M_{\infty}=0.00132\right)$ 


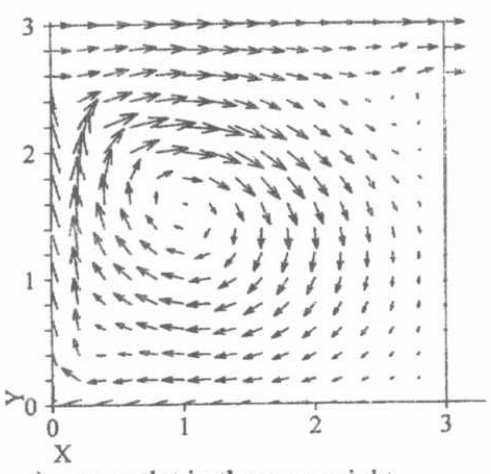

a) one outlet in the upper right corner of the leeward side wall

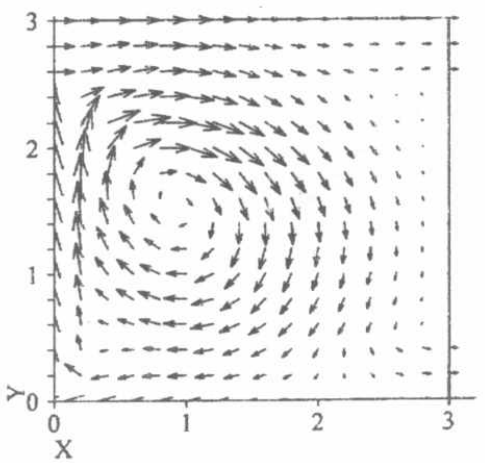

c) two outlets in the upper and lower right corners of the leeward side wall

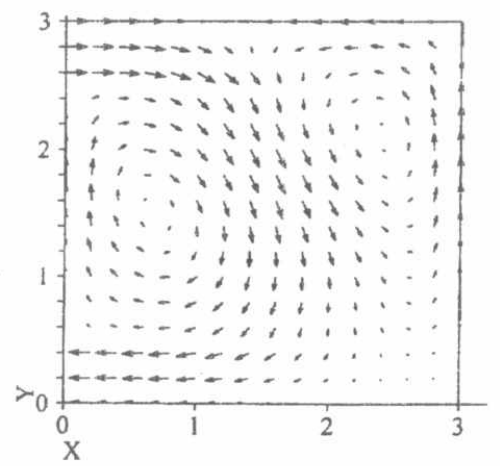

e) one outlet in the lower left corner of the upward side wall

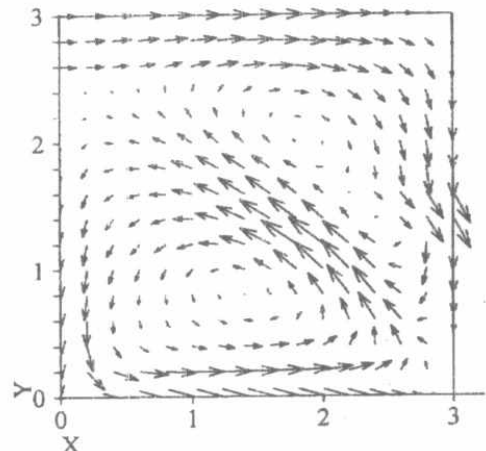

b) one outlet in the middle of the right leeward side wall

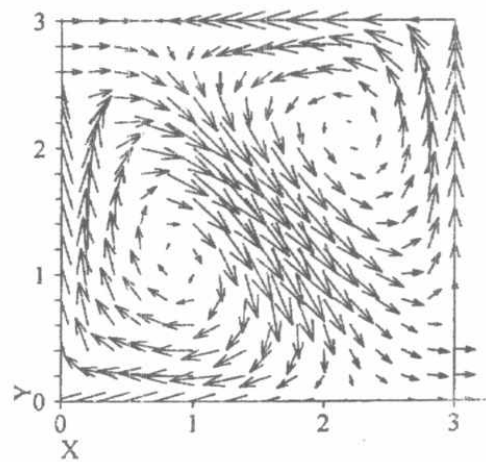

d) one outlet in the lower right corner of the leeward side wall

Figure 11 Effect of changing outlet location(s) on velocity vectors distribution inside chamber with $\mathrm{AR}=1\left(\Delta \mathrm{t}=0.06, \varepsilon=0.005, \mathrm{U}_{\mathrm{in}}=0.455 \mathrm{~m} / \mathrm{s}\right.$, i.e. $M_{\infty}=0.00132$ ) 

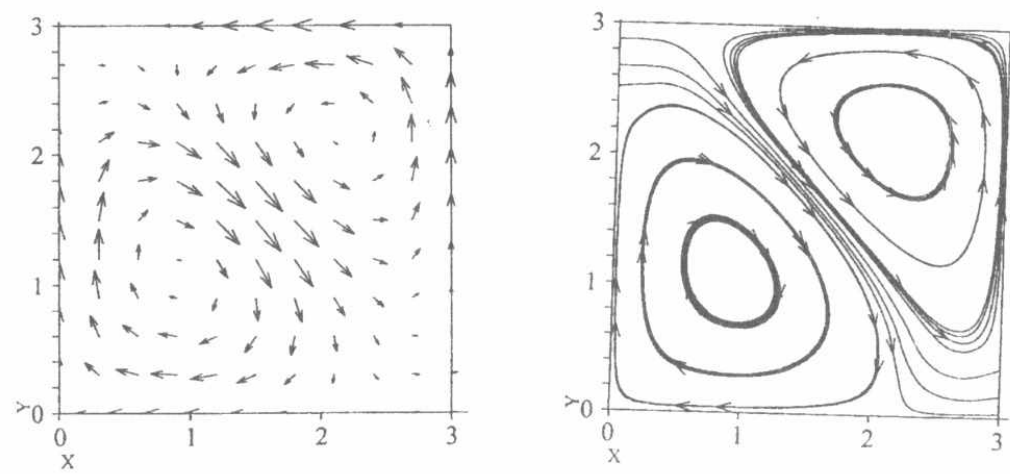

a) $21 * 21$ grid points
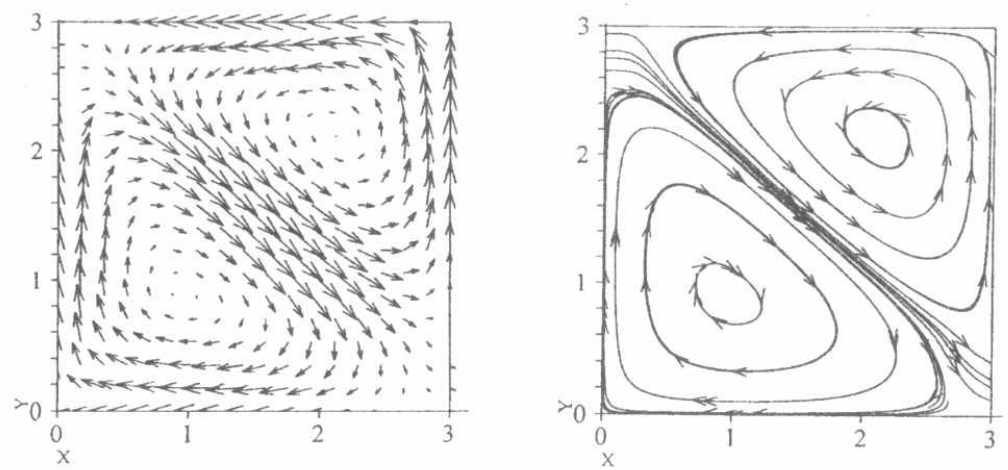

b) $35 * 35$ grid points
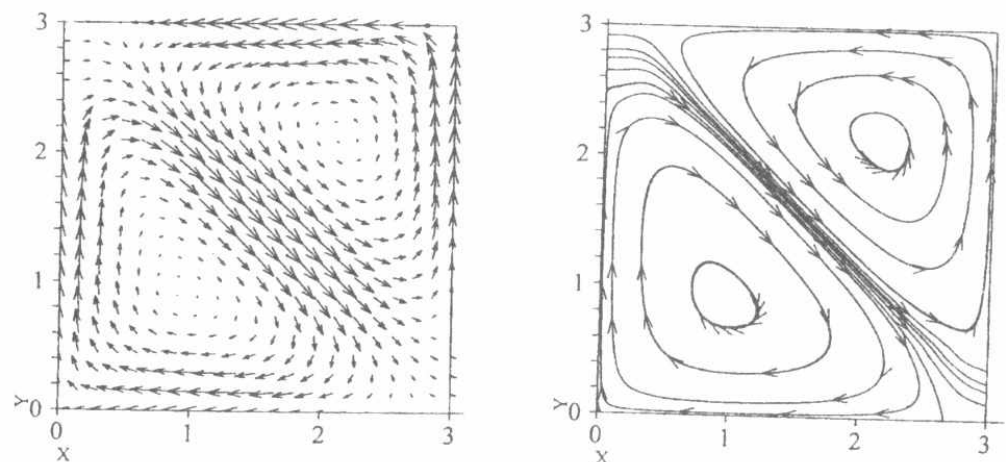

c) $41 * 41$ grid points

Figure 12 Effect of grid refinement on velocity vectors and streamlines distribution inside a chamber of $\mathrm{AR}=1\left(\Delta \mathrm{t}=0.06, \varepsilon=0.005, \mathrm{U}_{\text {in }}=0.455 \mathrm{~m} / \mathrm{s}\right.$, i.e. $\left.\mathrm{M}_{\infty}=0.00132\right)$ 